\title{
Limitaciones del enfoque de gestión estratégica en el sector público
}

Fabricio Franco

Pontificia Universidad Católica del Perú

ffranco@pucp.pe

Este artículo examina las limitaciones que la gestión estratégica ha tenido en el sector público para mejorar el desempeño y promover resultados en diversas organizaciones estatales. El problema está más allá de las dificultades para medir el desempeño e identificar resultados con imparcialidad en el sector público, y se asocia con un débil enfoque acerca de la naturaleza de lo "público" y sobre cómo se implementan las políticas públicas en las organizaciones gubernamentales.

Se presentan las características de enfoque convencional de gestión estratégica y se analizan los problemas al importar acríticamente el modelo del sector privado sin reconocer el carácter político de las organizaciones públicas y de su contexto, y que sus resultados se inscriben en el marco de las políticas públicas. Asimismo, se afirma que la implementación de sus estrategias se da en el marco de redes organizacionales y que la distinción entre la formulación y la ejecución no es siempre nítida.

Palabras clave: gestión estratégica; gestión pública; mejora del desempeño; políticas públicas; implementación

Limitations of the Strategic Management Approach in the Public Sector

This article examines the limitations that strategic management has had in the public sector to improve the performance and promote results in various state organizations. The problem is beyond the difficulties to measure the performance and identify results with impartiality in the public sector; rather, it is associated with a weak vision about the nature of the "public" sphere, and with how public policies are implemented in government organizations.

The characteristics of the conventional approach in strategic management are presented, and the problems of importing acritically the private sector model without recognizing the political character of public organizations and their context, along with the public policy framework in which its results are inscribed, are analyzed. Likewise, the article claims that the implementation of their strategies happens in the organizational network context, and that the distinction between formulation and execution is not always clear.

Key Words: strategic management; public management; performance improvement; public policies; implementation 


\section{Introducción}

La gestión estratégica ha sido un instrumento estándar en la caja de herramientas del sector público en los últimos 30 años, aunque en América Latina su uso es más reciente. Sin embargo, su utilidad para impulsar la gestión de las organizaciones públicas y el logro de resultados no ha sido claramente demostrada (Bryson y otros 2010; Poister y otros 2010). Algunas dificultades sobre cómo definir y medir el desempeño en el sector público y el carácter multidimensional de los factores que afectan la mejora del desempeño son indudablemente parte de la explicación. No obstante, un débil enfoque teórico acerca de la naturaleza de lo público y sobre cómo se implementan las políticas públicas en las organizaciones gubernamentales es subrayado como el problema principal del enfoque convencional en gestión estratégica.

Tratando de entender esta aparente debilidad, el presente trabajo analiza primero las principales características de esta herramienta y la promesa implícita en su aplicación con respecto a las mejoras organizacionales, en particular el establecimiento de las decisiones institucionales de largo plazo. A continuación, se evalúan los principales factores que pueden explicar una débil correlación entre el uso de este instrumento y el aumento del rendimiento en las organizaciones públicas (OP). La tercera sección analiza cómo el enfoque convencional tiene fallas en algunos supuestos básicos tomados del sector privado y se proporciona un punto de partida alternativo. Las secciones siguientes analizan a su vez las principales dificultades de este enfoque en el momento de la implementación. Finalmente, la conclusión resume los principales hallazgos.

\section{Las promesas y debilidades de una herramienta}

El enfoque convencional en gestión estratégica enfatiza la naturaleza secuencial del proceso reconociendo tres grandes fases/etapas: planificación/formulación, implementación y evaluación. En la inicial, los críticos primeros pasos dedicados al análisis del entorno externo e interno permiten la definición de los objetivos y metas de una OP' (Bryson 2011; Moore 2000). Esta importancia de la planificación es bien sintetizada en la famosa definición de Bryson, que dice que ella es: "un deliberado y disciplinado esfuerzo para producir decisiones y acciones fundamentales que den forma y guía sobre lo que una organización es, qué hace y por qué lo hace" (2011: 7-8). El supuesto es que esta etapa establece la trayectoria de los esfuerzos de la organización hacia el logro de las metas, proveyendo consistencia a las acciones y considerando el impacto de las restricciones externas e internas en su desempeño (Boyne y Walker 2010: 186).

En este sentido, la planificación sería la base reflexiva de la gestión estratégica que une un plan con su implementación en la organización, ampliando la atención al monitoreo de la ejecución, la gestión presupuestal, la medición del desempeño y la evaluación

\footnotetext{
1. En dicho enfoque hay dos aproximaciones alternativas en la secuencia de la planificación. En la primera, se realiza inicialmente un análisis del entorno interno y externo para luego definir o ajustar la misión y su dirección (misión); mientras que en el otro se parte de la definición estratégica para seguidamente analizar el entorno. Como se verá más adelante, en el sector público estas definiciones surgen en el contexto de la OP, en el marco de redes de políticas públicas que operan bajo lógicas políticas.
} 
(Poister y Streib 1999; Poister y otros 2010). Aplicado correctamente este proceso, fortalecería dos dimensiones esenciales interconectadas: fortalecer la capacidad de la organización y mejorar el desempeño, especialmente de aquellos vinculados al impacto final de su acción (Poister y otros 2010; Poister y Streib 2005).

En la primera dimensión, estarían incluidos elementos como fortalecer la relación con los actores involucrados (Rainey 2009; Boyne and Walker 2004); promover un liderazgo efectivo (Bryson 2011); atraer mayor apoyo político a la organización (Bryson 2012; LleweIlyn y Tappin 2003); mejorar el proceso de toma de decisiones (Walker 2013; Bryson 2011) y el pensamiento estratégico (Bryson y otros 2010; Lietdtka 1998). En la segunda dimensión, están directamente envueltos la mejora de los bienes y servicios públicos al optimizar su calidad, la satisfacción de los beneficiarios o el aumento de la productividad (Fukuyama 2014; World Bank 2012).

Sin embargo, aunque el uso de la gestión estratégica ha crecido desde la década de los 80 de la mano de las reformas de la Nueva Gestión Pública (NGP), introduciendo conceptos de mercado y prácticas para mejorar el gobierno (Pollitt y Sand 2011; Dunleavy y otros 2006), su repercusión en el desempeño organizacional está débilmente correlacionado (Poister y otros 2010). En otras palabras, su impacto en el desempeño no tiene una validación consistente y verificable entre diferentes tipos de OP. Los datos obtenidos principalmente a través de encuestas de percepción a autoridades locales británicas (Boyne y Walker 2010; Walker y otros 2010; Meier y otros 2010; Andrews y otros 2009) y gobiernos locales estadounidenses (Poister y Streib 2005) no son concluyentes ${ }^{2}$.

\section{Algunas causas que explican una relación elusiva}

Cuán elusiva es la relación entre la gestión estratégica y el desempeño organizacional en el sector público está determinado por factores entrelazados. El primero es la inherente dificultad para definir y medir el desempeño; el segundo es el carácter multidimensional sobre el que se fundamenta la mejora del desempeño; y, finalmente, un malentendido conceptual en el enfoque convencional de gestión estratégica con dos dimensiones interrelacionadas. Este último es el objeto central del presente artículo.

Ciertamente, un aspecto del problema es la variedad de opciones y la ambigüedad que tiene la medición del desempeño en el sector público (Moynihan 2008). Aquí no se dispone de indicadores discretos asociados con la participación de la empresa en la cuota de mercado o el beneficio obtenido para los accionistas. Variados desafíos surgen si la información del desempeño en la OP se relaciona con la medición de los impactos esperados, productos, la eficiencia (productividad) o con insumos tales como presupuesto, cantidad de personal, equipamiento, etc. (Heinrich 2012).

La complejidad de estos desafíos se vincula a si la información para la planificación estratégica está basada en datos administrativos o en encuestas, midiendo niveles de sa-

2. Como Hendrick (2010: 223) sugiere, estas encuestas que investigan el uso y el impacto de esta herramienta no permiten precisar si realmente se hace una aplicación sistemática y rigurosa del método de planificación estratégica o si son someros ejercicios de FODA de una sesión. Estudios en profundidad basados en estudios de caso son poco frecuentes y no recogen la experiencia de diferentes OP operando en contextos diversos. En español no se han encontrado estudios sistemáticos que establezcan una relación entre mejora del desempeño y el uso de la herramienta en el sector público. 
tisfacción de actores internos o externos; y si están focalizados en eficiencia o efectividad (Andrews y otros 2010). Walker y otros (2015) sugieren que marcadas diferencias en las percepciones pueden ser observadas entre funcionarios de la organización, los ciudadanos o reguladores. La controversia entre los actores involucrados con diversos intereses alcanza aún la definición misma acerca de qué indicadores son útiles para medir qué resultados. Van Dooren y otros (2010) y Rainey y Jung (2015) argumentan que estas características son inherentes a la dificultad para evaluar "objetivamente" los servicios y la acción pública.

Por otro lado, la mejora del desempeño depende de la comprensión sobre qué factores causales, además de la planificación, están asociados a esta. Sin embargo, no existe una forma sencilla de separar los efectos de varios factores interrelacionados. Algunas variables se relacionan con el contexto institucional en el que opera una OP, mientras que otras están asociadas a variables internas. A menudo, ambas están fuera del control de los directivos de la organización en el mediano plazo y, en consecuencia, del ámbito de aplicación de una planificación estratégica.

Las primeras son, por ejemplo, el nivel de apoyo de las autoridades políticas del gobierno con respecto a los objetivos de la OP y los recursos que normalmente tienen asignados (Poister y otros 2010); cuán centralizada es la estructura y relaciones de poder en el Estado en la que opera la OP (O'Toole y Meier 2015); la intensidad de las demandas y expectativas ciudadanas frente a temas del sector o la OP; la velocidad e impredictibilidad de los cambios de contexto sectorial en el que actúa (O'Toole y Meier 2015; Andrews 2009) ; y la diversidad y número de actores involucrados que participan en la política pública vinculada a la OP.

Las variables internas están asociadas a cuán centralizadas o jerárquicas son sus estructuras organizativas; a si la cultura del trabajo es receptiva a los cambios y está orientada a resultados (Boyne y Walker 2010); a la complejidad de los procesos de trabajo (Boyne y Walker, 2004); a la mayor o menor presencia en la organización de funcionarios del servicio civil o profesionales (Echebarría 2007); y al nivel de rotación de personal que puede hacer perder experiencia vital (Walker y otros 2015).

Sin embargo, como se afirmó, los factores primarios que explican la débil correlación con el desempeño organizacional constituyen dos dimensiones propias de la gestión estratégica: a) una teoría débil con respecto a cómo funciona una OP como parte del Estado, que se analiza en las siguientes dos secciones; y b) un enfoque que concibe la formulación y ejecución como etapas separadas de la gestión estratégica y no como momentos entrelazados, interactivos de un proceso continuo, tratado en las secciones finales.

\section{Una herramienta sin un marco teórico apropiado}

La gestión estratégica es una herramienta basada en modelos lógicos que formalizan una relación causal entre el contexto y los insumos, recursos, procesos internos, productos e impactos que produce la acción de una $\mathrm{OP}^{3}$. Cuando esta información es recolectada y analizada permite tanto la generación de estrategias como un conocimiento significativo,

3. Estos modelos usualmente se presentan bajo la forma de diagramas que establecen una secuencia y una relación entre los elementos mencionados de la organización y su entorno. Un ejemplo se observa en el anexo. 
que permite "atar los cabos" estableciendo las conclusiones y cursos de acción necesarios (Mintzberg 1994).

Sin embargo, todos estos modelos tienen algunos supuestos comunes, que se pueden resumir en que las diferencias entre el sector público y el privado son triviales o, si son sustanciales, son restricciones que deben superarse moviendo la gestión pública a un enfoque de negocios como el del sector privado (Rainey 2009; O'Toole y Meier 2015). En este enfoque, la premisa es que existe un comportamiento organizativo genérico que permite pensar una teoría de las organizaciones de aplicación general que, históricamente, se ha centrado en aportes conceptuales del sector privado. No obstante, como señala Sayre, lo público y lo privado, en términos de organizaciones, "son iguales pero en aspectos poco importantes" (Allison 1994: 16). Stone y otros (1999) subrayan algunas diferencias clave ligadas a cómo se gobiernan las organizaciones, la definición de sus objetivos/misión y su desempeño, y los mecanismos bajo los que se financian.

Este enfoque convencional tiene un núcleo de cuatro supuestos estrechamente entrelazados. El primero remarca que la estrategia es una competencia entre organizaciones por recursos escasos que maximizan su ventaja competitiva en un juego de suma cero; mientras el segundo destaca que en dichas organizaciones los tomadores de decisiones son individuos racionales que definen objetivos entre un conjunto de opciones seleccionadas técnicamente (Worth 2014; David 2011; William y Lewis 2008; Eppink y De Wall 2001). En tercer lugar, la alineación de la organización a su entorno es percibida como un mecanismo de ajuste acorde con un patrón socioeconómico darwinista (Breslin 2011) requerido para sobrevivir y desarrollarse (Miles y Snow 1978; Porter 1996). Finalmente, es consustancial a esta perspectiva considerar como unidad primaria de análisis a la organización, tomando la idea del sector privado de que las empresas operan individualmente o como líneas de negocios dentro de corporaciones.

Ciertamente, este enfoque aplicado a las OP plantea algunas ambigüedades y contradicciones visibles. Por ejemplo, los bienes y servicios públicos entregados a menudo se analizan como si estuvieran "despolitizados" y solo se centraran en el logro de metas de gestión, como lo reflejan Llewellyn y Tappin (2003) y Joyce (1999). En este sentido, su rol con respecto a fortalecer/debilitar la democracia, la legitimidad y las relaciones de poder en las relaciones Estado-sociedad quedan fuera de una mirada focalizada en la economía y la eficiencia. Así, dimensiones relevantes para medir el desempeño de una OP como la equidad, la imparcialidad o su capacidad de respuesta con la que brindan sus bienes y servicios quedan excluidas (Christensen y Laegreid 2007).

Como Steward (2004) agudamente observa, dichos servicios públicos a menudo se entienden vagamente como enmarcados dentro de políticas públicas ${ }^{4}$ y desarrollándose en entornos políticos 5 . Más aún, sus objetivos se miran ocasionalmente como una función exógena determinada por "los deseos [y] los mandatos de las prioridades políticas del go-

\footnotetext{
4. Es la adaptación del concepto de "policy" que no tiene una traducción directa al español y que es a veces confundida con "política" que es un concepto polisémico en español. Aquí política pública se entiende como la acción gubernamental desarrollada para dar solución a un asunto público (Guerrero 1993).

5. El término política se utiliza aquí en un sentido más restringido, para referirse a la práctica del poder dentro de la administración pública, en términos de la toma de decisiones, su implementación, financiamiento y las medidas tomadas para su aplicación efectiva.
} 
bierno de turno" (Walker 2013: 676) o, a veces, de forma endógena como las empresas lo hacen y como la anterior cita de Bryson nos recuerda.

Por ello, los estudios de caso evaluados con frecuencia se centran en agencias públicas, en particular en aquellas que tienen cierto grado de autonomía de la presión política y que brindan servicios cuasi públicos ${ }^{6}$ (Joyce 1999). Ello es especialmente notorio en la literatura especializada en inglés. Esta perspectiva es coherente con la NGP que destaca una clara separación de dimensiones política/política pública de la de administración, visualizando a los políticos como una interferencia para una gestión más técnica por parte de gerentes públicos (Pollitt y Sand 2011).

Este marco ha dado lugar a traducciones sobresimplificadas de estrategias del sector privado, como la aplicación del modelo de las cinco fuerzas de Porter (Vining 2011; Boehm 1996) o el de las competencias básicas (Worthington 2009), que poco aportan al pensamiento y acción estratégica en el sector público. Incluso modelos adaptados más cuidadosamente, como el concebido por Boyne y Walker (2004) y luego probado por Meier y otros (2010), Walker (2013), y Poister (2011), no han establecido vínculos importantes entre la gestión estratégica y el rendimiento en agencias estatales.

¿Están estos supuestos reflejando apropiadamente cuál es rol del Estado y cómo el sector público y sus organizaciones realmente operan? ¿Puede un alto funcionario público diseñar estrategias utilizando un marco analítico con taxonomías incoherentes derivadas del mundo de la empresa?

\section{5. "Público" es un adjetivo muy importante para las estrategias en el sector público}

El enfoque convencional descrito anteriormente es muy diferente al de pensadores clásicos como Wilson (1887), Weber (1964), Lasswell (1951), Lindblom (1979) y Waldo (1955). Todos ellos concuerdan en tres aspectos esenciales con respecto a las OP: i) la gestión pública está fuertemente vinculada al proceso político; ii) el proceso de las políticas públicas es una característica definitoria de las OP; y iii) estas organizaciones son elementos de una red, a veces fragmentada, llamada Estado y deben entenderse en ese contexto. Para desarrollar estas afirmaciones, que vinculan la gestión de las OP con las dimensiones política y de política pública, y luego abordar los cuatro supuestos citados de la gestión estratégica, se necesita aclarar previamente algunos conceptos básicos.

Las funciones básicas del Estado y su relación con la sociedad no tienen un carácter universal y pueden ser determinadas por las tradiciones administrativas -anglosajona, napoleónica, etc. (Painter y Peters 2010)- o por su nivel de intervención siguiendo modelos como el Estado de Bienestar o Estado Competitivo (Evans y Cerny 2003; Jessop 1999). Según varios autores (Davis y Moore 1945; Le Galès 2010; Fukuyama 2014), estas funciones serían la aplicación efectiva de las leyes, asegurar el bienestar y la seguridad a través de la prestación de servicios públicos, la regulación de la actividad privada y dirimir los intereses en conflicto en la sociedad.

6. Ejemplos comunes de bienes cuasi públicos son el uso de autopistas, el aseo urbano, las viviendas subvencionadas o servicios de salud y educación. 
Para cumplir con estas funciones son implementadas políticas públicas con el fin de abordar un problema de interés colectivo en un sector y/o territorio en particular, y son la materialización de un compromiso político que espera alcanzar ciertos resultados (Thoening 2009). Estos resultados -incluso los imprevistos- son el impacto de un conjunto de productos, en términos de bienes y servicios públicos, normas y reglamentos, producidos por una OP que opera individualmente o en una red de políticas públicas. Estas redes están principalmente compuestas por otras organizaciones públicas y actores privados y sociales con diferentes roles (Rhodes 1997).

Para darle soporte a dichas funciones, el aparato del Estado -una macro organización política- está integrado por una extensa red de OP que cumplen diferentes tareas (Jessop 1990), que incluyen más de 87000 en sistemas federales como el de Estados Unidos de Norteamérica (Raadschelders 2011: 84) y sobre las 2100 en sistemas unitarios de un país como Perú (PCM). Algunas están focalizadas en la provisión de bienes y servicios a los ciudadanos, regular organizaciones privadas, transferir recursos y subsidios, recolectar impuestos o supervisar la acción pública (Dunleavy 1989). Otras, tales como ministerios del nivel central o provincial/regional, están a cargo de diseñar políticas públicas, coordinando y supervisando su cumplimiento. En países donde el proceso de creación de agencias autónomas fue más radical -países anglosajones-, la división del trabajo entre aquellos que "dirigen y reman" es más clara, mientras que en el resto del mundo ambas funciones se superponen en los grandes y clásicos ministerios sectoriales.

El concepto de gobierno es el principio organizador que guía el funcionamiento de este conjunto de OP, entendido como "los procesos institucionales a través de los cuales decisiones colectivas vinculantes son tomadas dentro de un Estado-nación" (Heywood 2007: 19). Los gobiernos a su vez están modelados por estructuras constitucionales con sistemas federales o unitarios, y con regímenes políticos que pueden ser democráticos o autoritarios. En los democráticos, existen sistemas presidenciales y parlamentarios, teniendo los últimos mecanismos consensuales o de confrontación para procesar sus decisiones (Hendricks 2010).

Estas configuraciones políticas y organizativas que muestran la complejidad existente en cada país se desarrollan en diferentes sectores a diferentes ritmos y marcan decisivamente las estrategias en el sector público.

Ahora, teniendo un marco básico que describe cómo las OP operan dentro de un gobierno, se pueden abordar de mejor manera los supuestos de la planificación estratégica citados.

En primer lugar, parece desequilibrado basar solo en la competencia las estrategias públicas cuando, tanto la dinámica que les rodea como sus objetivos, están más cerca de los enfoques de colaboración y en consecuencia embarcados en juegos no de suma cero (Brown 2010). Por ejemplo, ¿operan en un entorno competitivo y en busca de una ventaja competitiva una oficina de impuestos internos como la Superintendencia Nacional de Administración Tributaria (SUNAT) o el Ministerio de Justicia y un Gobierno Regional? En el sector público, tratando de resolver problemas societales, probablemente son necesarios marcos teóricos que expliquen concurrentemente enfoques colaborativos (cooperativos) y competitivos (Lichbach 1996). Para una OP, la cooperación frecuentemente es una estrategia para obtener ventaja política, conseguir recursos adicionales, resolver objetivos de política pública o simplemente útil para obedecer un mandato legal (Weiss 
1987). Evidentemente, una OP que provee bienes cuasi públicos y funciona en un contexto comercial necesita estrategias competitivas, pero simultáneamente puede jugar con reglas más cooperativas con otros actores gubernamentales que son también parte de su entorno inmediato ${ }^{7}$.

En segundo lugar, no es habitual que la gestión estratégica, en cuanto a los procesos de toma de decisiones internas, conduzca a una OP a la definición de objetivos de largo plazo como en el mundo privado (Steward 2004). La ley define el propósito de las organizaciones gubernamentales y sus énfasis específicos son delineados por políticas públicas establecidas por actores políticos con esta función dentro del aparato estatal (los ministerios, el poder legislativo, un alcalde en su competencia, etc.) y son la expresión de una acción colectiva enmarcada por una racionalidad limitada (Simon 1972).

Aquí los objetivos organizacionales y los objetivos de política pública son la encarnación de las demandas sociales procesadas y negociadas por el sistema político, siguiendo a menudo un mecanismo incremental (Lindblom 1979), en lugar de individuos racionales dentro de una organización. Esto no es contradictorio con que algunos parámetros de los bienes y servicios como calidad, costo y beneficiarios, estén sujetos a negociación de la OP y sus directivos con un ministro y/u otros actores dentro de una red de políticas públicas.

En cuanto al tercer supuesto, es bueno preguntarse si el nivel apropiado de análisis para la gestión estratégica en el sector público es siempre una OP individualmente concebida. Si el comportamiento de una organización está influido por las oportunidades y limitaciones del contexto sobre sus objetivos y sus variables internas (Johns 2006), en este sector las dimensiones política y de política pública son determinantes en la gestión estratégica y éstas funcionan en el marco de redes.

Habitualmente, la estrategia de una OP es la consecuencia de una política pública, la relación política con su red de actores y aquellos organismos estatales que controlan su acción y luego de su equipo directivo. Estas relaciones tienen lugar en el marco de una estructura de poder político concentrada o dispersa que es relevante para la definición de una estrategia con respecto a la negociación de recursos y fondos, así como rendir cuentas a diferentes instancias supervisoras. Por tanto, sistemas unitarios o federales, parlamentarios o presidenciales, tienen un impacto en el grado de fragmentación de la estructura de poder y los procesos de aprobación y supervisión que marcarán la evolución de la estrategia de una OP (O'Toole y Meier 2015: 242).

Por consiguiente, una mejor perspectiva para la comprensión del proceso de la estrategia en el sector público sería un nivel intermedio de análisis, en lugar de centrarse en una organización, que solo es una vista parcial de un fenómeno más complejo. Un enfoque más integral, entendiendo que las políticas públicas se forman y se implementan en redes de organizaciones (Devereaux y otros 2011; Johansson 2014) que operan dentro estructuras políticas y procesos de aprobación determinados, sería más útil para pensar estratégicamente cursos de acción en el sector público en general y en una OP, en particular.

7. De hecho, estas lógicas también operan en el sector privado, por ejemplo en las alianzas globales entre aerolíneas o la nueva organización productiva de las cadenas globlales de valor. 
Dicho rápidamente, la estrategia de una organización gubernamental está generalmente subsumida por una mayor, con dimensiones asociadas a políticas públicas, y opera bajo una lógica política. Tal cosa no existe en el sector privado y, por tanto, requiere otro marco analítico.

Finalmente, la alineación de una OP al contexto se puede entender como la búsqueda de legitimidad formal dentro de la red de políticas públicas en la que opera y sus instancias políticas de control, y no obligatoriamente en búsqueda del acceso a mayores recursos. Al respecto, su meta sería la alineación del impacto de su acción o de sus servicios con los valores y expectativas de las autoridades políticas y actores de su red de políticas públicas (Stillman 1974). Si esto no se logra, una opción estratégica es mostrar que la OP está en camino de desarrollar las capacidades para alcanzarlos (Andrews 2013). Los "malos" resultados en políticas públicas tienen un impacto sobre el sistema político y, a través de instancias como los ministerios o legislaturas, esto se traslada sobre la OP concernida, sus altos funcionarios y su estrategia. Las OP no quiebran, pierden legitimidad.

Si estas afirmaciones fueran correctas, el rendimiento y la estrategia general de una organización estatal estarían relacionados con tres estrategias específicas interconectadas y que podrían ser mejor estudiadas desde un nivel de análisis intermedio: una estrategia política, una estrategia de política pública y una estrategia organizacional. Sin embargo, esta perspectiva está completamente ausente del enfoque convencional en el uso de la herramienta y refleja el déficit en la teorización de la gestión estratégica en el sector público.

\section{La implementación no es automática ni su contingencia}

La segunda dimensión sobre la que el enfoque convencional presenta insuficiencias es su perspectiva sobre la implementación. El punto aquí es: ¿se puede concebir la implementación como una fase separada, operando en una secuencia lineal a la formulación y teniendo lugar predominantemente dentro de una OP?

La bibliografía en gestión estratégica (David 2011; Boyne 2011) entiende la implementación como una "traducción" de la estrategia que, reconociendo las particularidades de la estructura organizativa, el sistema de control interno y la cultura organizativa, ejecuta actividades que apuntan a alcanzar los objetivos y metas formuladas. Esta etapa es considerada la más compleja ${ }^{9}$ del proceso de gestión estratégica y conlleva habitualmente cambios organizacionales en la o las OP "implementadoras." Estos pueden ser de naturaleza diversa, como ajustes a los procedimientos de gestión vigentes, a los instrumentos de política pública utilizados, a la introducción de nuevas tecnologías, a la modificación del sistema de monitoreo o arreglos a diversos aspectos inter o intraorganizacionales. Dichos cambios, en última instancia, afectan cómo los funcionarios actúan individualmente y colectivamente o cómo las organizaciones interactúan; en otras palabras, introducen un cambio en el propio

8. Ver por ejemplo Boyne (2011: 286-316) y Davis (2011: 210-248).

9. Considerada como la etapa más compleja, con mayores vaivenes y más prolongada es, paradójicamente, la que cuenta con el menor desarrollo conceptual tanto desde el enfoque convencional de gestión estratégica como en el de políticas públicas. Para un ejemplo simplemente gráfico, ver en anexo los modelos lógicos de Davis y Boyne en los que se observa un menor nivel de formalización y desarrollo conceptual de la etapa mencionada. 
comportamiento, práctica e interacciones de los implementadores (Banco Mundial 2012), así como en la del grupo objetivo de la política pública ${ }^{10}$.

Sin embargo, el elemento medular a subrayar es que la implementación incorpora nuevos actores, espacios de interacción y procesos decisorios que no necesariamente estuvieron envueltos en la formulación original de la estrategia (Meny y Thoenig 1992) y que, con frecuencia, son profundamente políticos. Esto produce eventualmente efectos no previstos en el enunciado de la política pública, en la formulación de la estrategia y sus resultados esperados. Su materialización es una serie de decisiones operativas y acciones que buscan hacer efectiva la decisión tomada por una autoridad política superior y en la que suelen interactuar también actores no gubernamentales (Winter 2012; Lester y Goggin 1998). Su ejecución transforma la intención de la política pública y su programación operativa en un conjunto de reglas, rutinas y procesos sociales soportados por recursos financieros que convierten la intención en acción: en bienes y servicios públicos y/o regulaciones. Este proceso es la esencia de lo que se denomina implementación (O’Toole 2012).

En el sector público es reconocido desde hace largo tiempo ${ }^{11}$ (Pressman y Wildavsky 1984) que los objetivos e intenciones originales de los decisores en el momento de la formulación se ven habitualmente alterados durante la implementación. Como señalan Winter (2012) y Grindle (2009), la brecha de implementación es la diferencia entre lo formulado, materializado en el marco normativo y su respectivo plan estratégico, y lo concretado en la práctica. A veces, esta brecha puede ser reducida o puede ser enorme, una de cuyas expresiones adversas más extremas es la no ejecución de un programa o proyecto público (Grindle 2009). Como sutilmente señala esta autora, esta divergencia no siempre es negativa, pero sí es normalmente imprevisible desde la perspectiva de aquellos que formulan el plan, sus metas, actividades y presupuestos.

¿Por qué surge esta brecha luego de haber realizado un ejercicio de planificación estratégica? Hay una diversidad de variables concernidas (Goggin y otros 1990) que pueden asociarse a tres grandes dimensiones sometidas a la contingencia: i) la falta de objetivos claros o valores congruentes en los mandatos de una OP y/o en la política pública a implementar que deja espacio para la interpretación; ii) la dificultad para entender el rol que juega la discrecionalidad y la motivación entre los funcionarios a cargo de su ejecución; iii) la importancia creciente que tiene en la implementación una larga cadena de interacciones entre múltiples actores en la producción y provisión de bienes y servicios (Mégie 2012; Grindle 2009; Barrett 2004).

Un cuarto elemento que no se considera aquí, ya que se asume la existencia de un proceso de planificación estratégica con una base técnica mínima, es la implementación de políticas con un sustento técnico débil y sin una relación causal sobre el problema público que se aborda. No obstante, y como lo señalan diversos estudios (Kaufmann y otros 2015; Banco Mundial 2010; Grindle 2009), este fenómeno es aún prevalente en la gestión de diversas políticas públicas en la región.

10. Considérese el efecto sobre la conducta de usuarios y ciudadanos como la introducción del uso del cinturón de seguridad o la regulación sobre la prohibición de fumar en recintos públicos.

11. Al menos desde inicios de los años 70, con la publicación original de Pressman y Wildavsky (1973). Aquí se consultó la tercera edición de 1984. 
Las dificultades del enfoque convencional para abordar estas tres dimensiones están asociadas con una perspectiva prescriptiva "de arriba hacia abajo" sobre cómo debería operar el Estado y sus organizaciones. Se remarca la distinción entre el "estamento" político que toma decisiones y uno administrativo que se encarga de ponerlas en operación basado en una visión piramidal y jerárquica en la relación entre las OP y al interior de estas (Weber 1964; Wilson 1887).

Aquí se privilegia la importancia de la toma de decisión y la formulación por sobre la implementación y se observa el proceso general de gestión como una secuencia lineal no sujeta a interacciones sustantivas hasta su finalización y evaluación ${ }^{12}$ (Hassenteufel 2009: 28-33). La puesta en operación sería el mundo de la racionalidad técnica y la selección de los instrumentos apropiados -cuando no exclusivamente de su aplicación- para los objetivos de política pública determinados. Además, siguiendo el modelo de Elección Pública ${ }^{13}$, supone que dichos objetivos y los instrumentos seleccionados son racionales y explícitos, lo que facilita el control del desempeño de los implementadores y de los resultados finales.

Así, la ejecución fluiría a través de una cadena de mando en automático en la que las eventuales desviaciones de los implementadores obedecerían a anomalías ligadas a la búsqueda de sus propios intereses corporativos: mayor presupuesto, promoción laboral, etc. (Fuenmayor 2014). Esta visión es consustancial con el modelo principal-agente y con las reformas de la NGP orientadas a sortear estas anomalías o divergencias a través de privatizaciones, la tercerización, la delegación a agencias públicas autónomas o subnacionales operando bajo esquemas gerenciales de medición del rendimiento (Gofen 2014; Pollit y Sand 2011).El problema es que la realidad no suele operar de esa forma. Normalmente, está sujeta a la contingencia de diversos eventos y el comportamiento de actores difíciles de anticipar plenamente en el momento de la formulación.

\section{Los factores, la contingencia y la brecha}

Existen tres factores centrales que sugieren un enfoque alternativo que conciba la formulación y la implementación como momentos interactivos y esencialmente políticos. Estos son la mencionada ambigüedad de mandatos institucionales y/o de los objetivos de política pública; los elementos que gatillan la discrecionalidad de los implementadores; y la emergencia estructuras de implementación con múltiples actores.

Es habitual en el sector público la presencia de mandatos institucionales y objetivos de política pública ambiguos e incluso conflictivos que condicionan el alineamiento entre la estrategia formulada y su implementación. Casos como el Sistema Nacional de Evaluación, Acreditación y Certificación de la calidad Educativa (SINEACE) ${ }^{14}$, un organismo regulador cuyas atribuciones están sujetas a debate por diferentes actores, reflejan la difi-

12. Su fundamentación está en los trabajos de Lasswell (1951) y Jones (1970).

13. Este modelo se sustenta en la Teoría de la Elección Racional y la Escuela Neoclásica.

14. Sistema Nacional de Evaluación, Acreditación y Certificación de la Calidad Educativa, organismo autónomo del sector educación. 
cultad para instrumentar una política sobre la que existe aparentemente pleno consenso, como es mejorar la calidad de la educación ${ }^{15}$.

Como señalan Rainey y Jung (2015), este tipo de mandatos en las OP afectan tres aspectos de su gestión: dirigir consistentemente sus actividades; evaluar la calidad de su desempeño y establecer sus prioridades frente a los intereses divergentes de los actores involucrados. La hipótesis es que las OP con alternativamente altos niveles de control político, focalizados en atender complejos problemas y/o totalmente dependientes de recursos públicos tenderán a tener mayores niveles de ambigüedad o conflictividad en su misión y mandato.

Lo mismo sucede con políticas públicas que incorporan valores y componentes que son difíciles de conciliar, como el desarrollo de una "minería que privilegie el acceso al agua, la preservación del medio ambiente y la inclusión (...) social de las comunidades de su entorno" (MEM). Esta situación también se presenta en programas con objetivos que tienden a superponerse y entrar en colisión -conceptualmente y en el territorio- con aquellos en curso en otros sectores. La asignación de lotes petroleros (sector energía) en áreas naturales protegidas (sector medio ambiente) en la Amazonía es un buen ejemplo analizado por Alza (2009). Estos casos expresan los amplios márgenes de interpretación sobre el significado preciso de mandatos institucionales u objetivos de política pública que se abren asiduamente a funcionarios u OP implementadoras. Como se puede colegir, la interpretación en el nivel operativo de dichos mandatos institucionales y objetivos tiene a menudo consecuencias políticas en la implementación, divergiendo significativamente con la estrategia original formulada.

El segundo factor es el asociado al rol de la discrecionalidad y su motivación entre los funcionarios más próximos a las actividades de prestación de servicios. Este fenómeno es analizado desde hace más de 30 años (Lipsky 1980), aunque su impacto se complejizó en la década de los 90 con las reformas de la NGP, como se analizará más adelante.

Un punto aquí es si profesores, enfermeras, doctores, policías, trabajadores sociales y sus respectivos supervisores en terreno comparten el mismo enfoque que los formuladores de las políticas públicas, tienen las prácticas e incentivos esperados o simplemente interpretan de la misma forma las intenciones de los primeros (Winter 2012). En términos generales, las causas sobre la discrecionalidad -en forma de adaptación, alteración o no ejecución de las actividades del plan estratégico- y la motivación subyacente de dichos funcionarios se organiza en torno a dos perspectivas vinculadas.

La primera subraya que es el producto inevitable de la ya anotada ambigüedad de los objetivos de algunas políticas públicas (Rainey y Jung 2015; Pressman y Wildasky 1984; Lipsky 1980; Brodkin 2003) que produce decodificaciones particulares por los implementadores. Esta perspectiva está directamente conectada al concepto de racionalidad limitada de Simon (1972). Ejemplos sobre cómo interpretar, materializar y evaluar políticas que buscan "crear empleo digno" o "fomentar la inclusión de niños con habilidades diferenciadas en la educación regular" suponen, más que seguir una instrucción o cumplir con un indicador. Aquí lo determinante es

15. Un ejemplo de objetivos ambiguos es el referido a la función clave del Ministerio del Ambiente (MINAN) que señala su responsabilidad para "coordinar la implementación de la Política Nacional Ambiental con los sectores, los gobiernos regionales y los gobiernos locales" Ministerio del Ambiente (MINAN), pero no establece criterios específicos sobre los debería sustentarse esta coordinación horizontal y vertical. 
la aplicación de un criterio adecuado por los implementadores, que es bastante más complejo que un protocolo para colocar una vacuna o una multa de tránsito (Hupe y otros 2014).

Sin embargo, su carácter ineludible es consecuencia también de las diferentes percepciones, contextos institucionales y limitada información compartida entre formuladores e implementadores de las políticas públicas (Hupe y otros 2014; Keiser 2010; Hupe y Hill 2007). En esta línea argumentativa, el concepto de la cultura organizacional subyacente y las prácticas de gestión de las OP implementadoras son un elemento determinante (Burnes 2014).

La segunda perspectiva enfatiza que la divergencia es una elección deliberada que realizan estos funcionarios en base a una preferencia racional que maximiza su interés (Brodkin 2011; Ricucci 2005), como resultado de una elección ética que busca favorecer a los beneficiarios de un programa (Gofen 2014; Loyens y otros 2010) o en base a un criterio profesional/personal al percibirse que la política entra en contradicción con este (Gofen 2014; Tummers 2011).

En esta línea, hay estudios empíricos recientes en materias como educación, salud primaria, salud mental, bienestar social que cuestionan algunos de los supuestos básicos del modelo principal-agente en implementación (Gofen 2014, 2013; Hupe y otros 2014; Brodkin 2011, 2007; Tummers 2011). En ellos, se sugiere que variables como el sector y programa específico, el contexto institucional (Ministerio del Gobierno Central, Gobierno Regional o Municipalidad) y la profesión de los funcionarios pueden dar lugar a motivaciones diferenciadas (Gofen 2014; Hupe y otros 2014) no atribuibles a la búsqueda de preferencias corporativas.

En este sentido, parece inherente a diversas políticas la discrecionalidad de los implementadores, y limitados los resultados por controlarlos usando los mecanismos del modelo burocrático clásico o los adaptados de la práctica empresarial. Ni más reducidos tramos de control (más jerarquía), la estandarización de procedimientos o una mayor atención sobre insumos del primero, ni la delegación, la tercerización o las privatizaciones del segundo parecen haber eliminado el problema.

En América Latina ambas modalidades han sido experimentadas, aunque las vinculadas a la NGP han tenido mayor importancia en diversas políticas y programas estratégicos en los últimos años. Un ejemplo al respecto, es la experiencia temprana del presupuesto por resultados con paquetes cerrados de productos y herramientas de intervención centralmente definidos para su implementación por diversos actores. Este tipo de aproximación, formulada por ministerios de hacienda ha terminado con frecuencia desdibujando roles y responsabilidades en la ejecución por parte de actores sectoriales y subnacionales sin alcanzar los resultados esperados (Kaufmann y otros 2015; Banco Mundial 2010).

Como afirman estudios longitudinales, programas sociales y los mecanismos de la NGP por subordinar la discrecionalidad a los objetivos de la política pública terminan generando los mismos problemas bajo nuevas formas (Brodkin 2007). Así, el ritualismo burocrático en el cumplimiento de normas no está muy alejado del cumplimiento formal de indicadores de desempeño estipulados en convenios de gestión, contratos o en las "buenas prácticas" que dan lugar a formas de isomorfismo mimético ${ }^{16}$ (Brodkin 2011). De esta

16. Ver especialmente Pritchett y otros (2012) y Andrews (2013). 
manera, no existen mecanismos plenamente efectivos para regular discrecionalidad de los funcionarios que son la cara visible de las políticas públicas y que terminan generalmente dándole su forma final.

El tercer factor que condiciona la implementación es la larga cadena de interacciones que con frecuencia se requieren para la provisión final de ciertos bienes y servicios públicos. Aquí suelen intervenir diferentes OP, organizaciones privadas y sociales no solamente con intereses divergentes sino también con rutinas de trabajo y lenguajes técnicos disímiles, y con enfoques diferenciados sobre cómo abordar los objetivos de política pública (Peters 2014; O’Toole 2012). Esto da lugar a mecanismos de coordinación y al desarrollo de estrategias de colaboración que conduce a que varios actores públicos jueguen un rol central, especialmente por la necesidad de alinear intereses, estilos de gestión y culturas organizacionales diferentes.

Este contexto institucional fue parcialmente gatillado por las reformas liberales de las décadas 1980 y 1990 que reconfiguraron el rol del Estado y los mecanismos de operación gubernamental. Fundamentalmente, se separaron la función política de la operacional, desconcentrando la acción hacia niveles subnacionales o agencias autónomas y promoviendo una mayor confianza en el mercado y en políticas basadas en instrumentos de mercado (Saetren 2005; Barrett 2004). Ello generó progresivamente redes de políticas públicas que no sólo inciden en la formulación y diseño de las políticas públicas, sino que también participan en su puesta en operación. Con el tiempo, estas interacciones han constituido relaciones estables creando estructuras (Peters 2014) o contextos inter organizacionales de implementación definidos que desafían la capacidad de articulación y coordinación de los formuladores de las políticas públicas (O’Toole 2012).

Básicamente, existen tres tipos de estructuras, según los actores que participan y el tipo de interacción que tiene lugar. En la primera, un programa gubernamental opera completamente dentro de los márgenes del sector público en una configuración horizontal o vertical. En la horizontal, las OP del mismo nivel de gobierno son responsables de diferentes componentes, en términos de productos y actividades, en un mismo programa público. En este marco, la implementación involucra fundamentalmente mecanismos de coordinación que pueden complicarse si las OP son de diferentes sectores y por tanto responden a distintas autoridades políticas. En el esquema vertical o multinivel se articula un ministerio del nivel central con varios gobiernos regionales y/o locales. Aquí funcionan otros mecanismos de coordinación (Charbit 2011) o se presentan los problemas clásicos de ejecución cuando opera una jerarquía administrativa respondiendo a presiones contrapuestas de autoridades funcionales y territoriales.

Un segundo tipo de estructura está marcado por la externalización de actividades de un programa público hacia actores privados cuya lógica de funcionamiento opera bajo la dinámica de principal-agente (Miller 2005) en la que los incentivos contractuales y financieros son definitorios. Una tercera estructura son redes con organizaciones de la sociedad civil que asumen una forma similar a la del sector privado. Sin embargo, en este caso, los actores no gubernamentales pueden estar interesados en el área de la política pública res- 
pectiva -género, salud, educación, etc.- pero no compartiendo el mismo enfoque sobre el programa público en el que actúan como implementadores.

No obstante, si bien estas estructuras o su combinación producen arreglos institucionales por medio de contratos, convenios de gestión y acuerdos programáticos y presupuestales, es evidente que están lejos de operar de forma armoniosa. Es aquí justamente donde se observa la tensión entre lógicas competitivas, pero también colaborativas en las estrategias de los actores públicos de las redes de políticas. En la práctica, en algunos programas públicos de carácter nacional que combinan las tres estructuras señaladas, es frecuente la existencia de más de un actor central tales como un ministerio sectorial, el Ministerio de Hacienda, el Congreso o gobernadores regionales ejerciendo funciones de coordinación o supervisión. Estos escenarios complejos hacen difícil pensar la implementación como un proceso controlado y programado en su plenitud ex ante por un formulador central y racional, como lo sugiere el enfoque convencional (David 2011; Boyne 2011).

Ejemplos que reflejan los retos que enfrentan estas estructuras de implementación son programas de educación como el de "Acompañamiento Pedagógico"; programas de infraestructura como el "Programa Provías Descentralizado" del Ministerio de Transportes y Comunicaciones, o programas sociales como "Qali Warma" del Ministerio de Desarrollo e Inclusión Social.

Ciertamente, esto no condiciona la necesidad de una formulación estratégica y programación relativamente centralizada con respecto a quién hace qué, cómo y cuándo, pero sí subraya la importancia de incorporar sustancialmente las perspectiva de los ejecutores y las restricciones efectivas que enfrentan. Por otro lado, lo anterior no pone en cuestión el hecho de que la implementación esté sujeta a la contingencia propia de sistemas complejos y, consecuentemente, una interacción profunda entre formuladores e implementadores sea imprescindible. Ello puede conducir a veces a ajustes profundos en la puesta en operación y al desarrollo de mecanismos simbólicos (normativos y políticos) y de gestión (prácticos) para que la planificación no termine siendo un enunciado formal (Peters 2014: 136-137). En otras palabras, el proceso de implementación es un proceso político sujeto a contingencias que condicionan la forma final de las políticas públicas como producto de los factores analizados.

Por ello, Barrett (2004) sugiere que la implementación debe ser mirada como integral al proceso de las políticas públicas y como continuo al momento de la formulación, más que como un seguimiento administrativo. En ese sentido, la implementación puede conducir a la reformulación de la estrategia que la originó y, en ocasiones, a la redefinición de los objetivos mismos de la política pública. Lejos de ser un proceso lineal, la gestión estratégica sería con frecuencia un proceso interactivo, con reiteradas "idas y vueltas" entre la formulación y la implementación. Esta influencia recíproca -que no excluye mecanismos de control- puede comprender procesos de negociación política (Barret 2004), la construcción de discursos legitimadores y mecanismos de comunicación para alienar la lógica e intereses de diversos actores (Shanahan y otros 2011), pero también mecanismos de coordinación multinivel para integrar prácticas de gestión diferentes (Charbit 2011; OECD 2009). Sin estos mecanismos que acoplen a aquellos que buscan poner la política pública en efecto y aquellos de quienes depende la acción de hacerla efectiva, la brecha entre lo formulado y lo ejecutado será grande. 


\section{Conclusiones}

La aplicación de la gestión estratégica es parte de la ortodoxia en la gestión pública en las últimas décadas. Se trata de un proceso que promete impactar positivamente en el desempeño de las OP, fortalecer su capacidad para fijar objetivos y proporcionar consistencia a su acción considerando las oportunidades y restricciones de su entorno. Sin embargo, los resultados parecen no ser del todo concluyentes. Reconociendo las dificultades para definir y medir el desempeño en el sector público, así como los múltiples factores involucrados en la mejora del desempeño, se enfatiza que el principal problema de este instrumento es de orden conceptual en el enfoque convencional prevaleciente. Este problema tiene dos dimensiones: una débil teorización sobre cómo opera una OP dentro del Estado y un enfoque lineal y secuencial sobre su proceso de implementación.

Con respecto a la primera dimensión, se sostiene que existe una perspectiva basada en cuatro supuestos básicos que es generalmente aplicada para desarrollar estrategias asumiendo que las OP y su contexto son similares a aquellos del sector privado. En consecuencia, la complejidad en la forma como se definen las características políticas del contexto de las OP es normalmente soslayada. Se subraya la importancia de pensar estrategias en el sector público en términos de lógicas tanto competitivas como colaborativas que son la expresión de acciones colectivas que operan dentro de las redes de políticas públicas. En este sentido, el desempeño y la formulación de la estrategia de las OP sería más comprensible desde un nivel de análisis intermedio que la vincule con tres dimensiones: política, de política pública y organizacional.

Con respecto a la segunda, se analiza que el enfoque convencional percibe a la formulación y la implementación como etapas separadas de la gestión estratégica y en la que la ejecución opera como una traducción en automático de la planificación y centrada en una OP. Se analiza que existen al menos tres dimensiones que introducen una alta contingencia en la implementación de los programas y proyectos públicos que origina que sean procesos políticos complejos y no un simple monitoreo administrativo. Por lo anterior, se sugiere que la implementación sea mirada como un proceso continuo e interactivo al de la formulación y en el que crecientemente participan diversos actores públicos, privados y sociales. 
anexo

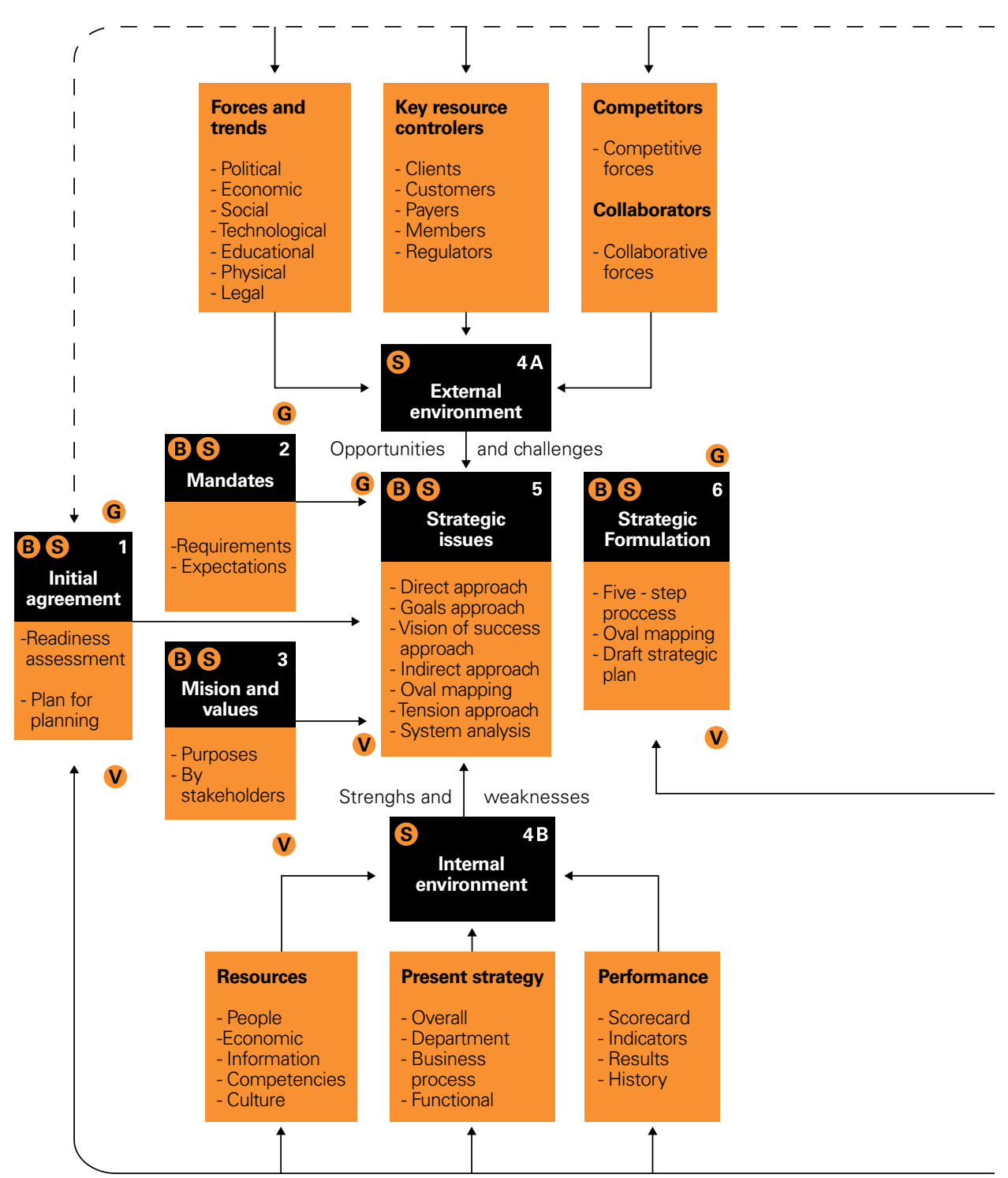


B = Places where the process typically begins

S = Places where stakeholder analyses may occur

G = Places where goal formulation may occur

V = Places where vision formulation may occur

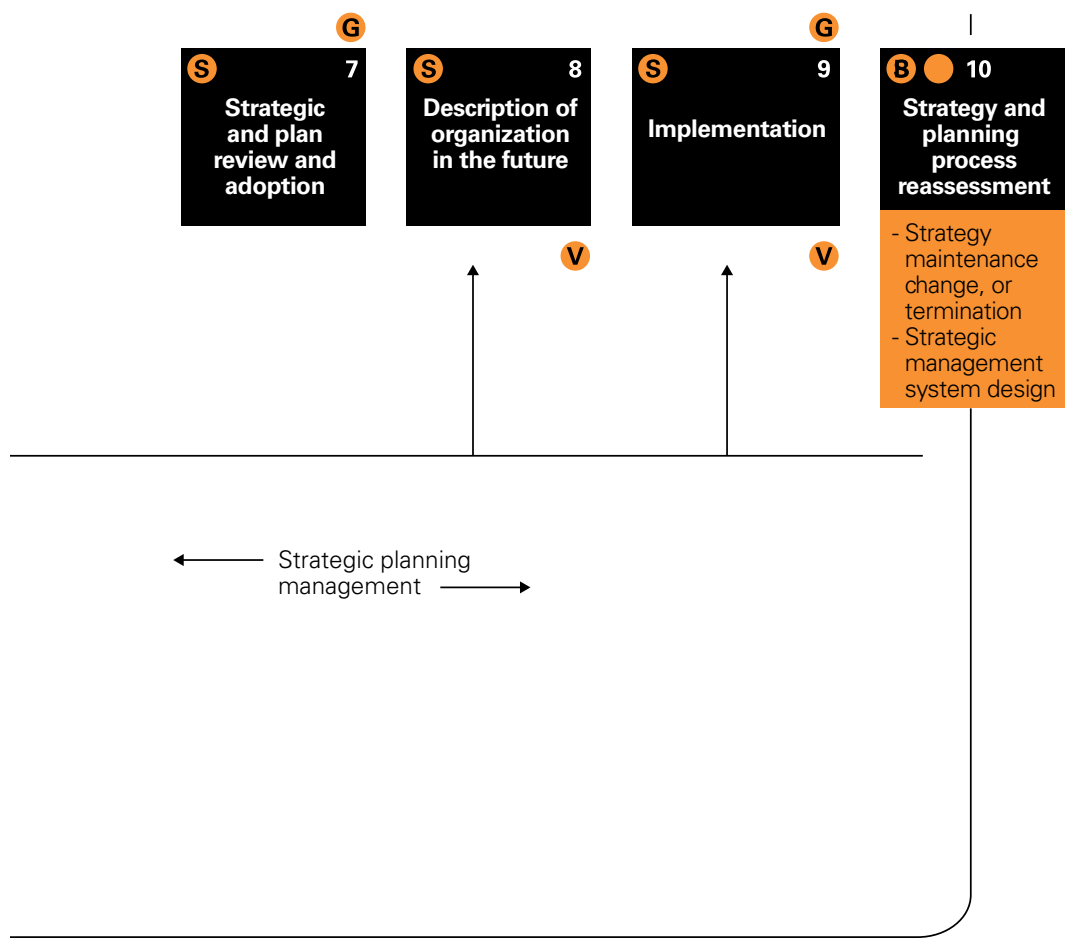


A comprensive strategic - management model

Chapter 10. Business ethics, social responsibility, and environmental sustainability

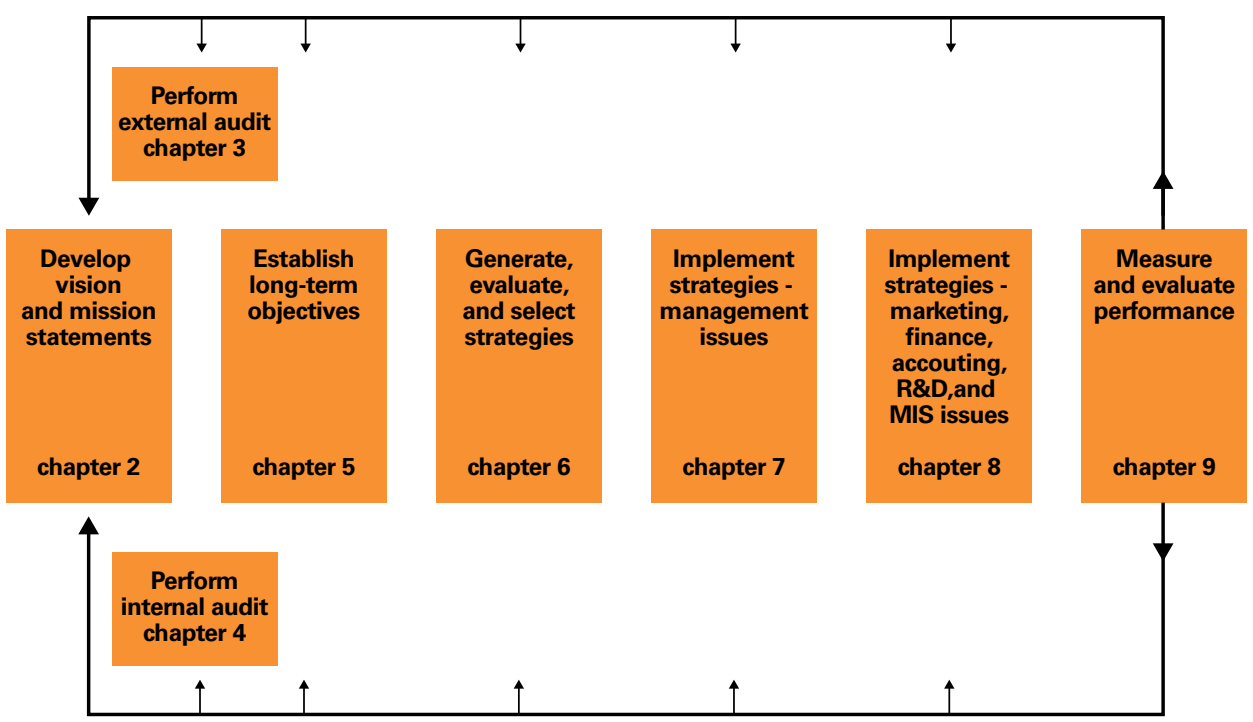

chapter 11: Global / international issues

Strategy

Strategy

implementation

Strategy

formulation

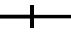

Source: Fred R. David, “How companies define their mission.” Long range planning 22, no. 3 (june 1988): 40. 


\section{bibliografía}

\section{Alza, Carlos}

2009

"Conflictividad en el Perú: una lectura desde las políticas públicas". En PEASE, Henry y Luis VILLAFRANCA (editores). Aula Magna 2008. Reforma del Estado: el papel de las políticas públicas. Lima: PUCP.

\section{Allison, Graham}

1994

"Public and Private Management: Are They

Fundamentally Alike in All Unimportant Respects?" En LAND, Frederick L. (editor). Current Issues In Public Administration. St Martin's Press, pp.16-32.

\section{Andrews, Matt}

The Limits of Institutional Reform in

Development. Changing Rules for Realistic Solutions. Cambridge: Cambridge University Press.

\section{Andrews, Rhys, George Boyne y Richard Walker}

"Strategy formulation, strategy content, and performance: An Empirical Analysis". Public Management Review. Volumen 11, número 1, pp. 11, 1-22.

\section{Andrews, Rhys, George Boyne,} Jae Moon y Richard Walker

"Assessing Organizational Performance:

Explaining Differences Detween Internal and External Measures". International Public Management Journal. Volumen 13, número 2, pp. 105-129.

\section{Banco Mundial}

2010

La formulación de las políticas públicas en la OCDE: Ideas para América Latina. Washington: Banco Mundial-LAC.

\section{Barret, Susan}

2004

“Implementation Studies: Time for a Revival? Personal Reflections on 20 Years of Implementation Studies". Public Administration Volumen 82, número 2, pp. 249-262

\section{Boehm, Amnon}

1996

"Forces Driving Competition in Human

Service Organizations and Positional

Competitive Responses". Administration

in Social Work, Volumen 20, número 4, pp. 61-78.

\section{Boyne, George y Richard Walker}

2004

"Strategy Content and Public Service

Organizations." Journal of Public Administration Research and Theory. Volumen 14, número 2, pp. 231-252.

\section{Boyne, George y Richard Walker}

2010

"Strategic Management and Public Service Performance: The Way Ahead". Public Administration Review. Volumen 70, suplemento S1, pp. 185-192.

\section{Breslin, Dermot}

"Reviewing a Generalized Darwinist Approach to Studying Socio-economic Change." International Journal of Management Reviews. Volumen 13, pp. 218-235.

\section{Brodkin, Evelyn}

"Bureaucracy Redux: Management Reformism and the Welfare State". Journal of Public Administration Research and Theory. Número 17, pp. 1-17.

\section{Brodkin, Evelyn}

under new managerialism". Journal of Public Administration Research and Theory. Número 21, pp. 253-77. 


\section{bibliografía}

\section{Brown, Trevor}

2010

"The Evolution of Public Sector Strategy".

Public Administration Review. Volumen 70, suplemento S1, pp. 212-214.

\section{Bryson, John}

2011

Strategic Planning for the Public and No profit Organizations. A Guide to Strengthening and Sustaining Organizational Achievements. Nueva Jersey: Jossey-Bass.

\section{Bryson, John}

2012

"Strategic Planning and Management". En GUY, Peters y Jon PIERRE (editores). The SAGE Handbook of Public Administration. Londres: Sage, pp. 50-63.

Bryson, John, Frances Berry y Kaifeng Yang

2010

"The State of Public Strategic Management Research: A Selective Literature Review and Set of Future Directions." The American Review of Public Administration. Volumen 40, número 5, pp. 495-521.

\section{Burnes, Bernard}

Managing Change. London: Financial

Times/Prentice Hall.

\section{David, Fred}

2011 Strategic Management: Concepts and Cases. Nueva Jersey: Pearson Education, Prentice Hall.

\section{Davis, Kinsley y Wilbert Moore}

"Some Principles of Stratification". American Sociological Review. Volumen 10, número 2, pp. 242-249.

\section{Charbit, Claire}

Working Papers, 2011/04, OECD Publishing. Consulta: 16 de febrero de 2016. http:// www.oecd.org/governance/regional-policy/48724565.pdf

\section{Christensen, Tom y Per Laegreid}

2007

Transcending New Public Management. Aldershot: Ashgate.

Devereaux, Jennings, Paul Zandbergen y Martin Martens

2011

"An institutional view of process strategy in the public sector", pp 492-518. En MAZZOLA, Pietro y Franz W. KELLERMANNS (editores). Handbook Of Research On Strategy Process, Cheltenham Eduard Elgar.

\section{Dunleavy, Patrick}

"The architecture of the British Central State, Part. 1: Framework for Analysis". Public Administration. Volumen 67, número 3, pp. $249-275$

\section{Dunleavy, Dunleavy, P. Marge-} tts, H. Bastow y J. Tinkler

"New Public Management is Dead - Long Live Digital Era Governance." Journal of Public Administration Research and Theory. Volumen 16, número 3, pp. 467-494.

\section{Echebarría, Koldo}

“El papel de la profesionalización del empleo público en América Latina". En Seminario Internacional sobre profesionalización del empleo público en América Latina. CIDOB. Barcelona.

\section{Eppink, Jan y Steven De Waal}

"Global Influences on The Public Sector." En SCHOLES, Kevan y Gerry JOHNSON (editores). Exploring Public Sector Strategy. Essex, FinancialTimes, Prentice Hall, pp. 38-56. 


\section{bibliografía}

\section{Evans, Mark y Philip Cerny}

2003

"Globalisation and Social Policy". En

ELLISON, N. y C. PIERSON (editores).

Developments in Social Policy 2. Londres:

Palgrave, pp. 19-40

\section{Fukuyama, Francis}

2014

Political Order and Political Decay: From

the Industrial Revolution to the Global-

ization of Democracy. Nueva York: Farrar,

Strauss and Giroux.

\section{Fuenmayor, Jennifer}

2014

"Política pública en América Latina en un contexto neoliberal: una revisión crítica de sus enfoques, teorías y modelos". Cinta de Moebio, revista epistemológica de Ciencias Sociales de la Universidad de Chile. Número 50.

\section{Gofen, Ana}

"Mind the Gap. Dimensions and Influence of Street-Level Divergence", Journal of Public Administration Research and Theory. Volumen 24, pp. 473-493.

\section{Goggin, Malcolm, Ann Bow-} man, James Lester y Laurence O'toole

Implementation Theory and Practice:

Toward a Third Generation. Glenview, III.: Scott, Foresman/Little,Brown.

\section{Grindle, Merilee}

"La brecha de la implementación". En MARIÑEZ, Freddy y Vidal GARZA (editores). Política pública y democracia en América Latina. Del análisis a la implementación. Ciudad de México, Instituto Tecnológico de Estudios Superiores de Monterrey, pp. 33-52.

\section{Guerrero, Omar}

1993

"Las políticas públicas como ámbito de concurrencia multidisciplinaria". Revista Ciencia. Volumen 44, número 1, pp. 29-40.

\section{Hassenteufel, Patrick}

2008

Sociologie Politique: I'action Publique.

París: Armand Colin.

\section{Heindrich, Carolyn}

“Measuring Public Sector Performance and Effectiveness". En GUY, Peters y Jon PIERRE (editores). The SAGE Handbook of Public Administration. Londres: Sage, pp. 32-49.

\section{Hendrick, Rebecca}

"What Is Wrong with Advice on Strategic Planning?". Public Administration Review. Volumen 70, pp. 222-223.

\section{Hendriks, Frank}

2010

Vital Democracy: A Theory of Democracy in Action. Oxford: Oxford University Press.

\section{Heywood, A.}

Politics. Basingstoke: Palgrave Macmillan.

\section{Hupe, Peter y Michael Hill}

"Street-level Bureaucracy and Public Accountability." Public Administration Número 85, pp. 279-299.

\section{Huper, Peter, Michael Hill y Monika Nangia}

2014 "Studying Implementation Beyond Deficit Analysis: The top-down View Reconsidered". Public Policy and Administration. Volumen 29, número 2, pp. 145-163. 


\section{bibliografía}

\author{
Jessop, Bob \\ 1990 \\ State Theory: Putting the Capitalist State in \\ Its Place. Cambridge: Polity. \\ Jessop, Bob \\ 1999 \\ "The Changing Governance of Welfare: Re- \\ cent Trends in its Primary Functions, Scale, \\ and Modes of Coordination." Social Policy \\ \& Administration. Volumen 33, número 4, \\ pp. 348-359.
}

\section{Johansson, Jan-Erik}

2014

"Strategic Governance in Public Agencies".

En JOYCE, Paul, John BRYSON y Mark

HOLZER (editores). Developments in

Strategic and Public Management: Studies

in the US and Europe. Basingstoke: Bas-

ingstoke Palgrave Macmillan, pp. 268-282.

\section{Johns, Gary}

2006

"The Essential Impact of Context on

Organizational Behavior." Academy of

Management Review. Volumen 31, número

2, pp. 396-408.

Jones, C.

An Introduction to the Study of Public Poli-

cy. Belmont (California): Duxbury Press.

\section{Joyce, Paul}

Strategic Management for the Public Services. Buckingham: Open University Press.

\section{Kaufmann, Jorge, Mario Sangi-} nés y Mario García

2015 Construyendo gobiernos efectivos.

Logros y retos de la gestión pública para resultados en América Latina y el Caribe. Washington: Banco Interamericano de Desarrollo.

\section{Keiser, Lael R}

2010

“Understanding Street-level Bureaucrats Decision Making: Determining Eligibility in the Social Security Disability Program". Public Administration Review. Número 70, pp. 247-257.

\section{Lasswell, $\mathbf{H}$.}

"The Policy Orientation". En LERNES, D. y H. LASSWELL (editors). The Policy Sciences. Stanford: Stanford University Press.

\section{Lester, James P y Malcolm Goggin}

"Back to the Future: The Rediscovery of Implementation Studies". Paper presented at the annual meeting of the American Political Science Association.

\section{Le Galès, Patrick}

2010

"Gouvernance". En BOUSSAGUET, Laurie, Sophie JACQUOT y Pauline RAVINET (editores). Dictionnaire de politiques publiques. París: Presses de Sciences Po, pp.299-308.

\section{Llewellyn, Sue Y Emma Tappin}

"Strategy in the Public Sector: Management in the Wilderness". Journal of Management Studies. Volumen 40, número 4, pp. 955-982.

\section{Lichbach, Mark} The Cooperator's Dilemma. Ann Arbor: University of Michigan Press.

\section{Liedtka, Jeanne M.}

"Strategic Thinking: Can It Be Taught?". Long Range Planning. Volumen 31, número 1, pp.120-129. 


\section{bibliografía}

\section{Lindblom, Charles E.}

1979

"Still Muddling, not yet Through". Public

Administration Review. Volumen 39,

pp. 517-526.

\section{Lipsky, Michael}

Street-level Bureaucracy: Dilemmas of the Individual in Public Services. Nueva York:

Russell Sage Foundation.

Loyens, Kim y Jeroen Maesschlck

2005

"Toward a Theoretical Framework for Ethical Decision Making of Street-level Bureaucracy". Administration \& Society. Número 42, pp. 66-100.

\section{Matthews, Jidith y Arthur}

\section{Shulman}

2010

"Competitive Advantage in Public Sector Organizations: Explaining the Public Good/ Sustainable Competitive Advantage Paradox". Journal of Business Research. Volumen 58, número 2, pp. 232-240.

\section{Mégie, Antoine}

"Mise en oeuvre". En BOUSSAGUET, Laurie, Sophie JACQUOT y Pauline RAVINET (editores). Dictionnaire de politiques publiques. París: Presses de Sciences Po, pp. 343-349.

\section{Meier, Kenneth, Laurence} O'toole, George Boyne, Richard Walker y Rhys Andrews

"Alignment and Results: Testing the Interaction Effects of Strategy, Structure, and Environment from Miles and Snow". Administration \& Society. Volumen 42, número 2, pp. 160-192.
Meny, Yves y

Jean-Claude Thoening

Las Políticas Públicas. Madrid: Ariel.

\section{Miles, R. E. y C. Snow}

1978

Organizational strategy, structure and process. Nueva York: McGraw-Hill.

\section{Ministerio del ambiente} (Minam)

Misión y Visión. Consulta: 17 de junio de 2015

http://www.minam.gob.pe/?el-ministeri$\mathrm{o}=$ mision-y-vision

\section{Ministerio de Energía} y Minas

$s / f$

Política Minera. Consulta: 17 de junio de 2015.

http://www.minem.gob.pe/_detalle. php?idSector=1\&idTitular=158\&idMenu= sub149\&idCateg $=158$

\section{Mintzberg, Henry}

"The Rise and Fall of Strategic Planning". Harvard Business Review. Volumen 72, número 1, pp. 107-114.

\section{Moynihan, Donald}

The Dynamics of Performance Management: Constructing information and Reform. Washington DC: Georgetown University Press.

\section{Moore, Mark}

"Managing for Value: Organizational Strategy in For-Profit, Nonprofit, and Governmental Organizations." Nonprofit and Voluntary Sector Quarterly. Volumen 29, número 1, pp. 183-204. 


\section{bibliografía}

OECD

OECD Policy Brief: Bridging the Gaps between Levels of Government. París: OECD Publishing.

\section{O'toole, Laurence y Kenneth} Meier

“Public Management, Context, and Performance- In Quest of a More General Theory". Journal of Public Administration Research and Theory. Volumen 25, pp. 237-256.

\section{O'toole, Laurence}

2015

"Interorganizational Relations and Policy Implementation." En GUY, Peters y Jon PIERRE (editores). The SAGE Handbook of Public Administration.Londres: Sage, pp. 234-244.

\section{Painter, Martin y Guy Peters}

Tradition and Public Administration. Basingstoke: Palgrave Macmillan.

\section{Peters, Guy}

2010

"Implementation Structures as Institutions." Public Policy and Administration. Volumen 29, número 2, pp.131-144.

\section{Poister, Theodore y Gregory} Streib

"Strategic Management in the Public Sector: Concepts, Models, and Processes". Productivity and Management Review. Volumen 22, pp. 308-325.

"Elements of Strategic Planning and Management in Municipal Government: Status After Two Decades". Public Administration Review. Volumen 5, número 1, pp. 45-56.

\section{Poister, Theodore, David Pitts y Lauren Edwards}

"Strategic Management Research in the Public Sector: A Review, Synthesis, and Future Directions". The American Review of Public Administration. Volumen 40, número 5, pp. 522-545.

\section{Poister, Theodore, Lauren Ed-} wards y Pasha Obed

"The Impact of Strategy Content and Development on Performance in Public Local Agencies". Annual Conference of the Public Management Research Association, Maxwell School of Citizenship and Public Affairs, Syracuse University, Syracuse, NY, 2/4 June 2011

\section{Pollitt, C.Y S. Sand}

2011

The Impacts of the New Public Management in Europe: A Meta-analysis. Coordinating for Cohesion in the Public Sector of the Future COCOPS. 14 December 2011. http://www.cocops.eu/ publications/research-reports. (Accessed 5 April 2012).

\section{Popa, Ion, Comin Dobrin y Otros.}

2011

"Competitive Advantage in the Public Sector". Theoretical and Empirical Research in Urban Management. Volumen 6, número 4, pp. 60-66.

\section{Porter, M.}

2011 Competitive Advantage. Nueva York: The Free Press.

\section{Porter, Michael}

Review. Volumen 74, número 6, pp. 61-78. 
Presidencia del Consejo de Ministros (PCM) Lista de organizaciones del Poder Ejecutivo:

http://www.peru.gob.pe/directorio/ pep directorio poderes.asp?cod poder $=3$ (Accessed 6 April 2015).

\section{Pressman, Jeffrey y Aaron Wildavsky}

Implementation. How Great Expectations

in Washington are Dashed in Oakland. Berkeley: University of California Press.

\section{Pritchett, Lant, Michael Wool-} cock y Matt Andrews

“Looking Like a State: Techniques of Persistent Failure in State Capability for Implementation." United Nations University، World Institute for Development Economics Research. Working Paper, número 63.

\section{Raadschelders Jos}

Public Administration. The Interdisciplinary Study of Government. Oxford: Oxford University Press.

\section{Rainey, Hal G.}

Understanding and managing public organizations. San Francisco: Jossey-Bass.

\section{Rainey, Hal G. y Chan Jung}

"A Conceptual Framework for Analysis of Goal Ambiguity in Public Organizations". Journal of Public Administration Research and Theory. Número 25, pp. 71-99.

\section{Rhodes, Roderick} networks, governance, reflexivity, and accountability. Buckingham: Open University Press.

Riccucci, Norma M.

How Management Matters: Street-level Bureaucrats and Welfare Reform. Washington DC: Georgetown University Press.

\section{Simon, Herbert}

"Theories of Bounded Rationality". En MCGUIRE C. B. y R. Radner (editores). Decision and Organization. Amsterdam: North-Holland Publishing Company.

\section{Saetren, Harald}

"Facts and Myths about Research on Public Policy Implementation: Out-of-Fashion, Allegedly Dead, But Still Very Much Alive and Relevant". Policy Studies Journal. Número 33, pp. 559-582.

\section{Shanahan, Elizabeth A.,} Michael D. Jones y Mark K. Mcbeth

"Policy Narratives and Policy Processes".

Policy Studies Journal. Volumen 39, número 3, pp. 535-561.

\section{Stewart, Jenny}

"The Meaning of Strategy in the Public sector". Australian Journal of Public Administration. Volumen 63, número 4, pp. 16-21.

\section{Stillman, Peter}

"The Concept of Legitimacy". Polity. Volumen 7, número 1, pp. 32-56.

\section{Stone, Melissa, Barbara Bige- low y William Crittenden}

"Research on Strategic Management in Nonprofit Organizations: Synthesis, Analysis, and Future Directions". Administration \& Society. Volumen 31, número 3, pp. 378-423. 


\section{bibliografía}

\section{Thoening, Jean-Claude}

1999

"Gouvernance". En BOUSSAGUET, Laurie,

Sophie JACQUOT y Pauline RAVINET

(editores). Dictionnaire de politiques

publiques. París: Presses de Sciences Po,

pp. $420-427$.

\section{Tummers, Lars}

2010

"Comment expliquer la disposition des

professionnels publics à mettre en oeuvre les nouvelles politiques: le système de I'aliénation des politiques". Revue Internationale des Sciences Administratives. Volumen 77, número 3, pp. 575-604.

\section{Van Dooren, W., G. Bouckaert y}

\section{J. Halligan}

2010

Performance Management in the Public Sector. Oxon: Routledge.

\section{Vinning, Aidan R.}

"Public Agency External Analysis Using

a Modified "Five Forces Framework".

International Public Management Journal.

Volumen 14, número 1, pp. 63-105.

\section{Waldo, Dwight}

The Study of Public Administration. Nueva York: Random House.

Walker, Richard, Rhys Andrews, George Boyne, Laurence O'tooley Kenneth Meier

“Wakeup Call: Strategic Management, Network Alarms and Performance". Public Administration Review. Volumen 70, número 5, pp.731-741.

\section{Walker, Richard}




\section{bibliografía}

Worth, Michael

2014

Nonprofit Management: Principles and

Practice. Thousand Oaks: Sage.
Worthington, Nick

2009

"Core Competencies in the Public Sector".

Proceedings of the ICE - Municipal Engi-

neer. Volumen 162, número 1, pp.51-55.

Fecha de recepción: 19/08/15

Fecha de aceptación: 13/10/15 